\title{
La realcalinización y la extracción electroquímica de los cloruros en las construcciones de hormigón armado
}

\author{
LUCA BERTOLINI*, FABIO BOLZONI*, BERNHARD ELSENER* ${ }^{\star \star}$, PIETRO PEDEFERRI* y CARMEN ANDRADE* *
}

Fecha de recepción: 7-II-96

Fecha de aceptación: $25-\mathrm{V}-96$
( $\left.{ }^{\star}\right)$ Dipartimento di Chimica Fisica Applicata, Politecnico di Milano, Milano. IT ALIA ${ }^{* \star}$ Institut for Baustoffe, Werkstoffchmie und Korrosion, ETH Politécnica Federal de Zurich. SUIZA ${ }^{(* *)}$ Instituto "Eduardo Torroja", CSIC-Madrid. ESPAÑA
RESUMEN

Se presentan técnicas electroquimicas de realcanización y extracción de cloruros, desarrolladas con el fin de rehabilitar estructuras carbonatadas y que contengan cloruros. Se discuten y comparan los mecanismos y consecuencias de la electrólisis y la electromigración, asi como las condiciones electroquimicas en la superficie de las armaduras con los de protección catódica. Se comentan, también, los posibles efectos secundarios.

\section{SUMMARY}

Realkalisation and electrochemical chloride removal techniques, developed for rehabiliting carbonated and chloride-containing structures, are presented. Electrolysis and electromigration mechanisms and consequences as well as electrochemical conditions at the reinforcement surface are discussed and compared with cathodic protection ones. Furthermore, possible side effects are commented.

\section{INTRODUCCIÓN}

La realcalinización y la extracción electroquímica de los cloruros son dos técnicas de reparación de obras de hormigón armado dañadas por corrosión de armaduras. Se pueden aplicar respectivamente a estructuras carbonatadas o contaminadas por cloruros y pueden restaurar, en el hormigón en contacto con las armaduras, los valores de $\mathrm{pH}$ y de concentración de cloruros que permitan la repasivación del acero.

Ambas técnicas ya han superado la fase de laboratorio y mostrado su eficacia sobre diferentes estructuras reales, pero no se pueden considerar todavía de rutina porque algunos aspectos, como por ejemplo la eficiencia al variar el tipo de hormigón y la geometría de la estructura o los efectos secundarios a largo plazo, no son todavía completamente conocidos.

El esquema operativo de estas técnicas es igual al de la protección catódica (Fig. 1): se impone una circulación de corriente continua entre un adecuado dispositivo anódico, conectado al polo positivo de una fuente de corriente y las armaduras, conectadas al polo negativo, funcionando, por ello, catódicamente. Sin embargo, a diferencia de la protección catódica, que tiene que operar desde el momen- 


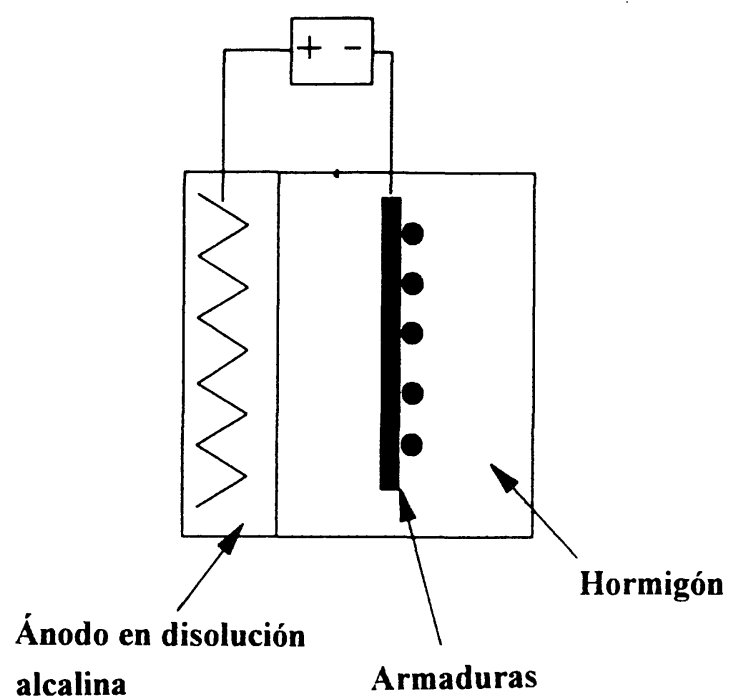

Fig. 1.- Esquema de funcionamiento de las técnicas de realcalinización y extracción electroquímica de los cloruros

to en que se aplica hasta al final de la vida de servicio de la estructura, los tratamientos de realcalinización y de extracción electroquímica de los cloruros duran un tiempo limitado (variando de una semana a tres meses). Consecuentemente, el dispositivo anódico, que en el caso de laprotección catódicaes parte integrante de la estructura, en el caso de la realcalinización y de la extracción electroquímica de los cloruros se aplica provisionalmente en el exterior de la misma, siendo "retirado" al finalizar la operación.

En la literatura se encuentran diferentes publicaciones sobre el tema [1-13]. En la redacción de ésta se ha hecho referencia, en particular, a [1] y [8] para los aspectos generales, y a [13] en lo que concierne a la comparación con la protección catódica.

\section{LAS MODIFICACIONES INDUCIDAS POR EL PASO DE LA CORRIENTE}

El paso de la corriente a través del hormigón entre el dispositivo y las armaduras provoca:

- disminución del potencial de las armaduras (se hace más catódico);

- reacciones electroquímicas en la superficie del ánodo y de las armaduras;

- electrodifusión (migración) de iones en la solución de los poros del hormigón.

Mientras la protección catódica proporciona, principalmente, los beneficios que tienen que ver con la disminución del potencial (ver [13]), las dos nuevas técnicas aprovechan los efectos de modificación química, inducidos por las reacciones anódicas y catódicas y por la electrodifusión.

\section{REACCIONES ELECTROQUIIMICAS EN LA SUPERFICIE DEL ÁNODO Y DE LAS ARMADURAS}

Las principales reacciones que se producen son las que inducen la electrólisis del agua, además de poder producirse otras reacciones en función, por ejemplo, del tipo de ánodo que se utilice. En base a la Figura 1, se pueden esquematizar los siguientes procesos.

\section{En el ánodo:}

La electrólisis del agua da lugar al desprendimiento de oxígeno, según una de las dos reacciones:

$$
\begin{aligned}
& 2 \mathrm{OH}^{-}-\mathrm{O}_{2}+2 \mathrm{H}^{+}+2 \mathrm{e}^{-} \\
& 2 \mathrm{H}_{2} \mathrm{O}-\mathrm{O}_{2}+4 \mathrm{H}^{+}+4 \mathrm{e}^{-}
\end{aligned}
$$

loque además provoca laacidificación del entorno (anolito). En medios alcalinos ( $\mathrm{pH}>10)$ prevalece la [1], mientras que es predominante la [1'] en $\mathrm{pH}<10$ (medios carbonatados).

Si además hay contaminación por cloruros, y el ánodo es muy polarizable (por ejemplo, titanio), entonces también se puede producir la formación de cloro gas, según:

$$
2 \mathrm{Cl}^{-}-\mathrm{Cl}_{2}+2 \mathrm{e}^{-}
$$

Este gas es conocido por su carácter fuertemente tóxico, por lo que no se podrá realizar una extracción de cloruros, por ejemplo, en lugares cerrados donde exista el riesgo de acumulación de este gas.

Además, si el ánodo no es de un material noble, se corroerá, según:

$$
\mathrm{Me}-\mathrm{Me}^{+\mathrm{n}}+\mathrm{ne}
$$

lo que dará lugar a la formación de óxidos, con cuya generación hay que contar al diseñar el conjunto del sistema.

\section{En el cátodo:}

La electrólisis del agua dará lugar a la formación de hidrógeno:

$$
2 \mathrm{H}_{2} \mathrm{O}+2 \mathrm{e}^{-}-2 \mathrm{OH}^{-}+\mathrm{H}_{2}
$$


Esta reacción es la que provoca, pues, la alcalinización del entorno de la armadura, fenómeno que igualmente se puede producir si se utilizan menores densidades de corriente (como la de la protección catódica), y sólo se alcanza a inducir la reducción del oxígeno atmosférico:

$$
1 / 2 \mathrm{O}_{2}+\mathrm{H}_{2} \mathrm{O}+2 \mathrm{e}^{-}-2 \mathrm{OH}^{-}
$$

Con relación a la reacción [4], es importante resaltar también que el hidrógeno puede producir unafragilización de la armadura, fenómeno irrelevante para el caso del hormigón armado, pero de efectos negativos en el caso del pre y postensado. Es por ello, por lo que no se recomienda aplicar estas técnicas en este tipo de estructuras.

Comparando las reacciones [1]/[1'] y [4] (que son las predominantes, en general, pues corresponden a la electrólisis del agua), puede deducirse fácilmente que, como el número de electrones intercambiados en el ánodo y cátodo debe ser el mismo, se producirá el doble de moléculas de hidrógeno que de oxígeno cuando la reacción predominante sea la [1'].

\section{PROCESOS INDUCIDOS POR LA CORRIENTE EN EL HORMIGÓN ENTRE ÁNODO Y CÁTODO}

Para que se produzca paso de corriente entre ánodo y cátodo, la carga debe ser transportada por el electrólito (hormigón). Para ello se produce el movimiento de los iones que existen en al fase contenida en los poros del hormigón bajo la acción del campo eléctrico. La carga es transportada:

- parte por los iones positivos que se mueven en la misma dirección del campo, es decir hacía el cátodo (en nuestro caso hacia las armaduras);

- y parte por los iones negativos que se mueven en dirección opuesta, es decir, hacía el ánodo (en nuestro caso se alejan de las armaduras).

La corriente total se puede expresar como suma de las corrientes transportadas por los diferentes iones:

$$
\mathrm{C}=\left(\mathrm{C}_{\mathrm{X} 1}+\mathrm{C}_{\mathrm{X} 2}+\mathrm{C}_{\mathrm{X} 3}+\ldots \ldots+\mathrm{C}_{\mathrm{X} 4}\right)
$$

donde $\mathrm{C}_{\mathrm{X}_{\mathrm{I}}}$ es la corriente transportada por la especie iónica $\mathrm{X}_{\mathrm{i}}$.

Se conoce como número de transporte $\mathrm{t}_{\mathrm{x}_{\mathrm{i}}}$ de la especie iónica $X_{i}$, la fracción de la corriente transportada esta especie, es decir:

$$
\mathrm{t}_{\mathrm{X}_{\mathrm{i}}}=\mathrm{C}_{\mathrm{Xi}_{\mathrm{i}}} / \mathrm{C}
$$

Este cociente depende, en primer lugar, del número de los portadores de la especie iónica Xi en disolución y, por tanto, de su concentración. En consecuencia, no contribuyen prácticamente al transporte las especies iónicas presentes en pequeñísimas concentraciones. Por ejemplo, en medios alcalinos, la fracción de corriente transportada por los iones $\mathrm{H}^{+}$es comparativamente despreciable, ya que es bajísima su concentración.

El número de transportedepende también de la movilidad, definida como la velocidad que la especie toma bajo la acción de un campo eléctrico unitario.

Desgraciadamente, en el caso del hormigón, no se conoce la movilidad de los diferentes iones presentes en la disolución, que podría depender, asimismo, de interacciones con las paredes, de una forma parecida a lo que ocurre en una disolución en el interior de una membrana. De cualquier forma, a efectos ilustrativos, en ausencia de datos mas precisos, se pueden considerar los datos de movilidad que se miden en las disoluciones y en condiciones de dilución infinita.

Para los iones de mayor interés para el transporte de corriente en el hormigón, la movilidad a dilución infinita de los siguientes iones es (en $\mathrm{cm}^{2} \mathrm{~s}^{-1} \mathrm{~V}^{-1}$ ):

$$
\begin{aligned}
& \mathrm{OH}^{-}=2,05 \times 10^{-3} \\
& \mathrm{Na}^{+}=5,2 \times 10^{-4} \\
& \mathrm{~K}^{+}=7,6 \times 10^{-4} \\
& \mathrm{Cl}^{-}=7,9 \times 10^{-4} \\
& 1 / 2 \mathrm{Ca}^{2+}=6,2 \times 10^{-4} \\
& \mathrm{HCO}_{3}=4,6 \times 10^{-4}
\end{aligned}
$$

Valores de números de transporte, cercanos y similares a estos, han sido estimados por Polder y Van Der Hondel [1], por Page [2] y por Andrade [16]. Polder valora, para morteros con $1 \%$ de cloruros (en peso con respecto al cemento) añadidos, como por ej. cloruro cálcico, aproximadamente 0,22 y 0,44 el número de transporte para $\mathrm{Cl}-\mathrm{yOH}^{-}$, respectivamente. Paraadiciones inferiores, tales que la proporción $\mathrm{Cl}-/ \mathrm{OH}^{-}$disminuye al valor crítico de 0,6 , el número de transporte de cloruros baja a 0,12 . Además, Polder, encuentra que el número de transporte varía con el perfil de concentración de cloruros, pasando de los estratos mas exteriores a los mas internos. Un hormigón (fabricado con cemento Portland), puesto en contacto por 6 meses con una disolución de cloruro sódico, presenta un número de transporte para los cloruros de $0,44,0,15$ y 0,07 , a profundidades de 10,20 y $30 \mathrm{~mm}$ respectivamente [1]. Page valora, para morteros con $2 \%$ decloruros (añadidos como $\mathrm{NaCl}$ ), el número de transporte del ion $\mathrm{Cl}^{-}$como 0,26 y 0,38 , para un cemento normal y un cemento sulfato-resistente (es decir, sin aluminato tricálcico), respectivamente. Andrade y colaboradores, en ensayos de migración, encuentran valores alrededor de $0,35-0,40[16]$. 


\section{Evolución de la composición de la fase acuosa de los poros del hormigón}

A continuación se analiza la disolución presente en los poros de hormigones en diferentes situaciones:

-Si el hormigón no está carbonatado ni tiene cloruros, esta disolución contiene iones $\mathrm{Na}^{+}, \mathrm{K}^{+}$y $\mathrm{OH}^{-}$(los iones $\mathrm{Ca}^{2+} \mathrm{y} \mathrm{SO}_{4}^{-}$se desprecian al estar en concentraciones muy pequeñas). Laaplicación de la corriente induce un aumento del contenido de álcalis en el hormigón $\left(\mathrm{OH}^{-} \mathrm{y}\right.$, por tanto, la de $\mathrm{Na}^{+}$y $\mathrm{K}^{+}$) desde una centena hasta algunas centenas de $\mathrm{mM} / 1 \mathrm{y}$, por tanto, el $\mathrm{pH}$ pasa de 13 a 13,5. Se puede, pues, asimilar esta disolución a una disolución alcalina constituida sólo por $\mathrm{NaOH}$, donde la corriente es transportada por lo iones positivos $\mathrm{Na}^{+}$y negativos $\mathrm{OH}^{-}$. Siendo las dos especies iónicas presentes iguales en concentración y la movilidad del $\mathrm{OH}^{-}$casi cuatro veces mayor que la del $\mathrm{Na}^{+} / \mathrm{K}^{+}$(el número de transporte de los $\mathrm{OH}^{-}$es 0,8 y el de $\mathrm{Na}^{+} / \mathrm{K}^{+}$es 0,2 ); en hormigón no carbonatado y no contaminadopor cloruros la contribución de los hidroxilos $\left(\mathrm{OH}^{-}\right)$al transporte de la corriente es, aproximadamente, cuatro veces mayor que la de los iones positivos $\mathrm{Na}^{+}$y K $\mathrm{K}^{+}$.

-En el caso de un hormigón contaminado por cloruros, la composición de la disolución en el interior de los poros depende del tipo y de la concentración de cloruros (por ejemplo de sodio o de calcio), pero también de la cantidad y del tipo de cemento utilizado (cloruros libres). Suponiendo que la sal contaminante sea cloruro sódico, una disolución típica, presente en los poros de un hormigón en condiciones de despasivar las armaduras, contiene una concentración de cloruros, al menos 0,6 veces la de $\mathrm{OH}^{-}$ (relación $[\mathrm{Cl}] /[\mathrm{OH}] \mathrm{0,6}$ ). Es decir, por ejemplo, para una concentración de contaminante $(\mathrm{NaCl})$, doble de la concentración de $\mathrm{NaOH}$ presente. La contribución al transporte de las diferentes especies podrá ser, por tanto:

para los hidroxilos $\left(\mathrm{OH}^{-}\right), \mathrm{n}^{\circ}$ transporte: $20,5 /(3 * 5,2+$ $20,5+2 * 7,9)=0,4$

para los cloruros, $\mathrm{n}^{\circ}$ transporte: $2 * 7,9 /(3 * 5,2+20,5+$ $2 * 7,9)=0,3$

$\mathrm{y}$, para el catión sodio, $\mathrm{n}^{\circ}$ transporte: $3 * 5,2 /(3 * 5,2+20,5$ $+2 * 7,9)=0,3$

Se puede notar, enseguida, cómo el aumento de $\mathrm{pH}$ en la superficie catódica será menor en ausencia de cloruros que en su presencia, ya que, en el primer caso, se aleja del cátodo el $80 \%$ de los $\mathrm{OH}^{-}$(número de transporte 0,8 ) producidos mientras que en el segundo caso sólo lo hace el $40 \%\left(n^{\circ}\right.$ transporte 0,4$)$.

Asimismo, se puede deducir, por lo que respecta a la eficacia del tratamiento, que sólo alrededor de un $30 \%$ de la corriente se invierte en extraer los cloruros.

-En el caso de hormigón carbonatado, su fase acuosa se puede considerar constituida por una disolución muy diluida de $\mathrm{pH}$ poco superior a 8 [17] que contiene, fundalmentalmente, álcalis $\left(\mathrm{Na}^{+}, \mathrm{K}^{+}\right), \mathrm{HCO}_{3}^{-} \mathrm{y} \mathrm{CO}_{3}$. Siendo la disolución próxima a la neutralidad, la concentración de los iones $\mathrm{H}^{+}$y $\mathrm{OH}^{-}$es de $10^{-7} \mathrm{~mol} / 1$, por lo que las especies que participen en el transporte de la corriente serán aquellas que estén en proporciones comparativamente superiores. Suponiendo que, por ejemplo, solamente los iones $\mathrm{HCO}_{3}^{-}$y $\mathrm{Na}^{+}$transportan la carga eléctrica, la contribución al transporte será:

para los iones $\mathrm{HCO}_{3}^{-}, \mathrm{n}^{\circ}$ transporte: $4,7 /(4,7+5,2)=0,48$

para los iones $\mathrm{Na}^{+}, \mathrm{n}^{\circ}$ transporte: $5,2 /(4,7+5,2)=0,52$

\section{Cuantificación del fenómeno}

Si bien una cuantificación rigurosa de la realcalinización producida no es posible sin delimitar bien la contribución de cada una de las reacciones que se pueden producir en paralelo en cada electrodo, a continuación se describirán ejemplos hipotéticos, para una mejor comprensión del fenómeno.

Todos los casos se esquematizan en las Figuras 2,3 y 4 , donde se ha supuesto que el ánodo es titanio y el cátodo la armadura, y que las únicas reacciones que se producen son las relativas a la electrólisis del agua (la [1'] y la [4])

Si suponemos que el proceso anódicoes el desprendimiento de oxígeno a partir del agua [1'] y el catódico el desprendimiento de hidrógeno [4]:

-en la superficie del titanio, 10 moléculas (por ejemplo) de agua producirán 5 moléculas de oxígeno y 20 iones $\mathrm{H}^{+}$, liberando, por tanto, a la fase metálica 20 electrones, según la reacción:

$$
10 \mathrm{H}_{2} \mathrm{O}=5 \mathrm{O}_{2}+20 \mathrm{H}^{+}+20 \mathrm{e}^{-}
$$

-mientras que en la superficie de las armaduras, 20 moléculas de agua (el doble que en el ánodo) con 20 electrones transportados por la fase metálica, producen 10 moléculas de hidrógeno y 20 iones $\mathrm{OH}^{-}$, según la reacción:

$$
20 \mathrm{H}_{2} \mathrm{O}+20 \mathrm{e}^{-}=20 \mathrm{OH}^{-}+10 \mathrm{H}_{2}
$$

Por lo tanto, distinguiendo en el hormigón una región anódica, una región catódica y una intermedia, como se esquematiza en las Figs. 2-4, se considerarán los tres casos siguientes. 
$\left.1^{\circ}\right)$ Se considera primero el caso de hormigón no carbonatado ni contaminado por cloruros que, en primera aproximación, por lo que concierne al transporte de la corriente, se asimila a una disolución diluida de $\mathrm{NaOH}$ (Fig.2). En la región anódica, por la reacción anódica entran 20 iones $\mathrm{H}^{+}$y por migración entran 16 iones $\mathrm{OH}^{-}$, mientras salen 4 iones $\mathrm{Na}^{+}$(no se tienen en cuenta los $\mathrm{H}^{+}$generados en el ánodo). En definitiva, el hormigón en este compartimento se acidifica. En la región intermedia, el número de las especies iónicas que entran compensa, exactamente, al de las que salen, por lo que no hay ninguna variación de composición. En la región catódica, por tanto, entran por migración $4 \mathrm{Na}^{+} \mathrm{y}$ salen $16 \mathrm{OH}^{-} \mathrm{y}$, debido a la reacción catódica, se producen 20 iones $\mathrm{OH}^{-}$. Por eso el hormigón se alcaliniza, ya que adquiere 4 moléculas de $\mathrm{NaOH}$.

$2^{\circ}$ ) En el caso de un hormigón que contiene cloruros (Fig.3), como se ha visto, la composición de la disolución en el interior de los poros depende de la concentración de cloruros pero, también, de la cantidad y del tipo de cemento utilizado, en particular de la presencia de constituyentes en condiciones de combinarse con los cloruros. Por ello, el número de transporte de los diferentes iones puede variar, pasando de un hormigón a otro, también, a igualdad de cloruros. De todas maneras, en referencia al caso antes descrito de hormigón contaminado por cloruros, con los números de transporte antes indicados, sólo el $40 \%$ de la acidez, o de la alcalinidad, producida en el ánodo o en el cátodo respectivamente se aleja de los electrodos (en ausencia de cloruros es el 80\%). Por eso, a igualdad de circulación de carga, el incremento de $\mathrm{pH}$ en el cátodo y su disminución en el ánodo, son mayores en presencia de cloruros con respecto a cuando no existen.

$3^{\circ}$ ) Finalmente, en el caso del hormigón carbonatado (en el esquema de la Fig. 4 se ha supuesto que la disolución presente en sus poros contenga sólo iones $\mathrm{Na}^{+}$y $\mathrm{HCO}_{3}^{-}$), el $50 \%$, aproximadamente, de la alcalinidad producida en la superficie de la región catódica permanece allí y el 50\% de la acidez producida en la red de titanio se queda en la región anódica, lo que contribuye a una más rápida reparación de la armadura.

Todos estos comentarios se pueden aplicar también para el cálculo del número de moles de especies generadas en los electrodos o transportadas a través del hormigón. Sin embargo, en los cálculos simples realizados, no se ha contabilizado que los procesos electródicos inducen unas variaciones de concentración que, a su vez, influyen en los valores de los números de transporte.

Así, si se considera el proceso de extracción de los cloruros, a medida que el proceso avanza, su número de transporte disminuye con su concentración, y también el rendimiento del proceso. Por ejemplo, Bennet y Schue [4]

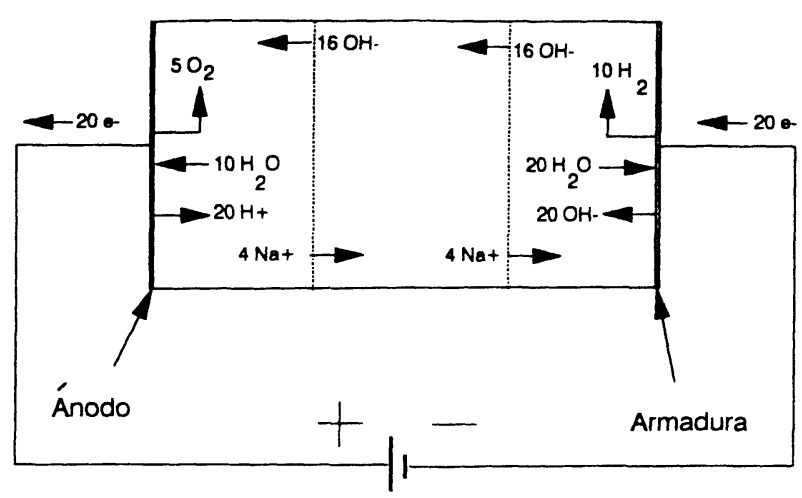

Fig. 2.-Esquema de las modificaciones que se producen en el hormigón no carbonatado y no contaminado por cloruros después del paso de la corriente entre el ánodo y las armaduras.

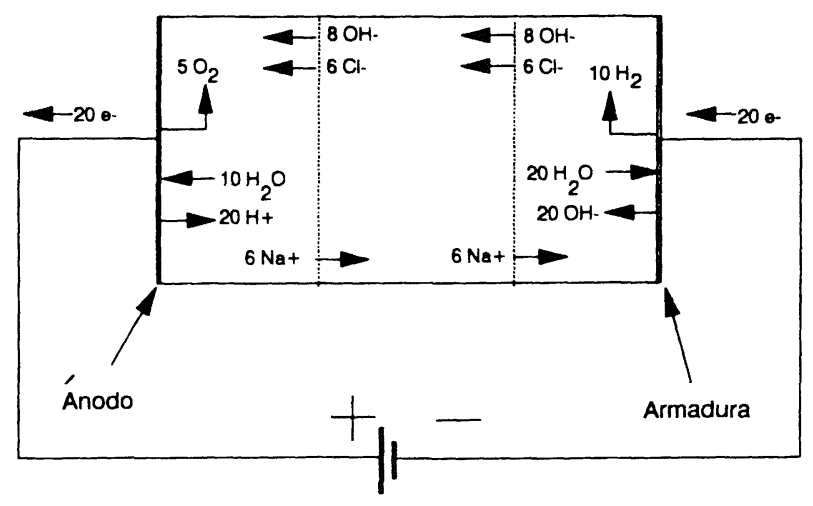

Fig. 3.- Esquema de las modificaciones que se producen en el hormigón contaminado por cloruros después del paso de la corriente entre el ámodo y las armaduras.

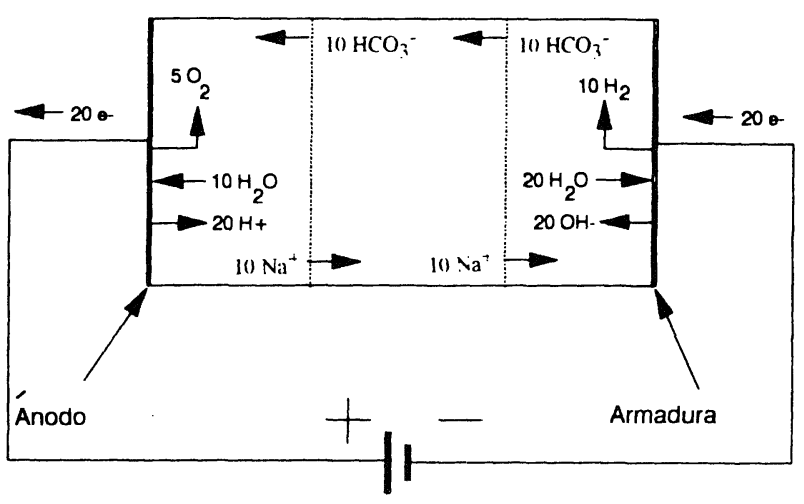

Fig. 4.- Esquema de las modificaciones que se producen en el hormigón carbonatado después del paso de la corriente entre ánodo y armaduras.

encuentran que la eficiencia del proceso baja de 0,4 a 0,06 (Fig.5). Este hecho es muy importante para la práctica de la extracción de cloruros: la eficacia de la extracción se va reduciendo a medida que su concentración disminuye cuando la extracción ha tenido lugar. 


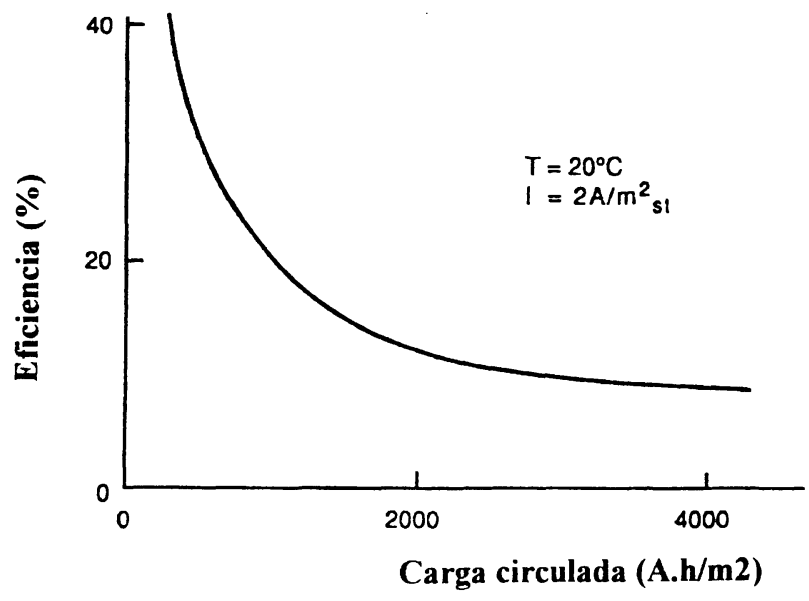

Fig. 5.- Eficacia de extracción de cloruros con respecto a la carga aplicada [4] durante un tratamiento de extracción de cloruros a $20^{\circ} \mathrm{C}$ y con una corriente de $2 \mathrm{~A} / \mathrm{m}^{2}$ (referida a superficie de armadura).

La cantidad de cloruros, extraída también, puede ser calculada teóricamente a partir de las ecuaciones clásicas de la electroquímica [16]. Para ello, se supone que la velocidad del cloruro se acelera por un factor que es proporcional a $\mathrm{zFDE} / \mathrm{RT}$ ( $\mathrm{z}=$ carga del cloruro, $\mathrm{F}=$ constante de Faraday, $\mathrm{R}=$ constante de los gases, $\mathrm{T}=$ temperatura absoluta, $\mathrm{D}=$ coeficiente de migración del cloruro $\mathrm{y}$ $\mathrm{E}=$-potencial aplicado). Además, se supone que la extracción se puede modelizar mediante la complementaria de la clásica solución de la ségunda ley de Fick, conocida como "la fórmula de la función de error" [18]. Es decir, el perfil de cloruros que se puede producir a lo largo del tiempo, suponiendo una concentración inicial homogénea a lo largo del hormigón, vendrá dado por:

$$
C_{x}=C_{i} \operatorname{erf}\left[\frac{x}{2 \sqrt{D t}}\right]
$$

donde $\mathrm{Cx}=$ concentración en el punto $\mathrm{x}, \mathrm{Ci}=$ concentración inicial y $t=$ tiempo.

\section{LAS CONDICIONES DE POTENCIAL, $\mathrm{pH} Y$ DE CONCENTRACIÓN DE CLORUROS EN LA SUPERFICIE DE LAS ARMADURAS Y LAS DIFERENCIAS CON LA PROTECCIÓN CATÓDICA}

\section{Estructuras contaminadas por cloruros}

En el diagrama potencial de armaduras/contenido de cloruros (expresado comoporcentaje en peso de los cloruros con respecto al cemento) de la Fig.6, se pueden distinguir diferentes zonas. Si el potencial es superior al de formación de picaduras (para E $>$ Epit) (punto 2), la armadura se encuentra en la zona en la cual el acero se corroe. Inmediatamente por debajo (Epit $>E>E$ Ero) (punto 3), se encuentra en la zona donde el ataque no se puede iniciar, pero puede avanzar si ya está iniciado. Más por debajo (E<Epro) (punto 8) está la zona donde la armadura se encuentra en condiciones de pasividad perfecta (es decir, la capa protectora, si la hay, es estable y, si no la hay o se destruye localmente, está en condiciones de reformarse). Por debajo del potencial de equilibrio del proceso de desprendimiento de hidrógeno (aproximadamente -900 $\mathrm{mV}$ vs $\mathrm{SCE}$ en el caso de $\mathrm{pH}=12$ ) (punto 5), está la zona donde es posible que se desprenda hidrógeno, por lo que si las armaduras son de acero de alta resistencia (como, por ejemplo, la de las estructuras pretensadas), o en el caso en el que el acero es susceptible de fragilización por hidrógeno (como por ejemplo algunos aceros templados y revenidos), este tipo de fragilización puede ocurrir. Finalmente, por debajo de $-1,1 \mathrm{~V}$ podemos indicar la zona donde es posible que tengan lugar fenómenos de reducción de adherencia entre armaduras y hormigón.

Así pues, la Fig. 6 ayuda a seguir la evolución de las condiciones en la superficie de las armaduras. El punto 1 indica las condiciones de la armadura que está en contacto con hormigón no contaminado. Cuando los cloruros han alcanzado la armadura, pero la concentración en su superficie no ha alcanzado el valor necesario para iniciar el ataque (indicativamente entre $0,4 \%$ y $1 \%$ con respecto al cemento), la armadura permanece pasiva y por eso el potencial es constante. Una vez que se alcanza el contenido crítico de cloruros (punto 2), el ataque corrosivo se produce y el potencial disminuye (punto 3). Suponiendo que antes de intervenir con el tratamiento de extracción de los cloruros pasa un cierto tiempo, la concentración de los cloruros crece posteriormente (punto 4).

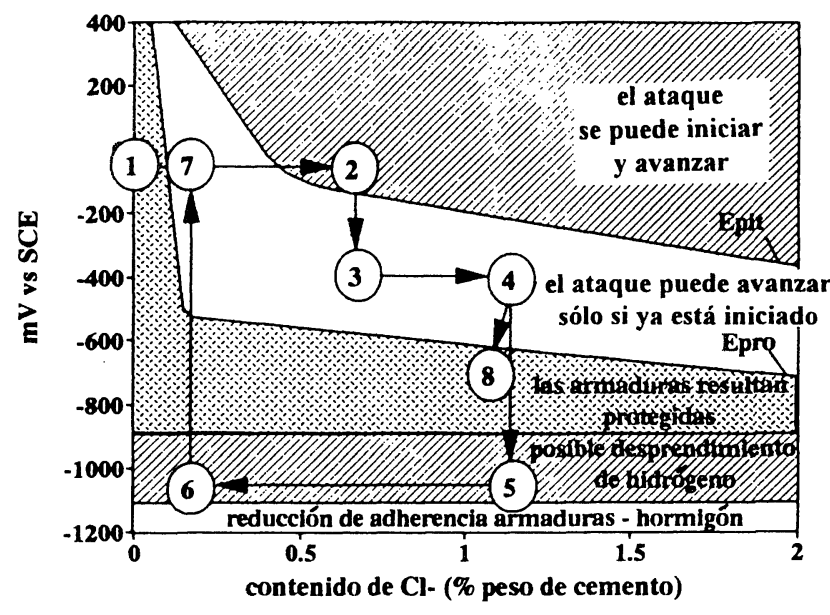

Fig. 6.-Condiciones depotencial y de contenido de cloruros en la superficie de las armaduras a partir de estructura nueva (1); en el momento en cl cual se inicia la corrosión (2); antes de activarse la extracción electroquímica de cloruros (4); durante el tratamiento (5-6) y con el tratamientoterminado(7). Para oomparación se indican también las condicionestípicas de laprotecoción catódica (8). 
Si se aplica protección catódica, la corrosión que ya está en marcha se puede bloquear, llevando el potencial de las armaduras a la zona de pasividad perfecta (punto 8) o, como más frecuentemente ocurre, se reduce solamente, disminuyendo el potencial en el intervalo (4-8) sin llegar al campo de pasividad perfecta. Para obtener estas disminuciones de potencial es suficiente imponer una circulación de corriente muy reducida (aproximadamente de 5 a $15 \mathrm{~mA} / \mathrm{m}^{2}$ [13] ). Consecuentemente, las variaciones del contenido de cloruros en el hormigón que rodea las armaduras (proporcionales a la corriente que circula) son modestas. Naturalmente, si se interrumpe la circulación de la corriente el ataque se reinicia o continúa, por lo cual se vuelve al punto 4 .

Si se aplica la extracción electroquímica de los cloruros, en este caso se impone una corriente muy elevada (de 2-3 ordenes de magnitud superior con respecto a la que se impone en la protección catódica) que aleja los cloruros de las armaduras. Por tanto, a partir del momento en el que se inicia la circulación de corriente, se pasa del punto 5 al punto 6 . Se alcanza este último después de algunas semanas de tratamiento. Una vez que el tratamiento está terminado, se alcanza el punto 7, donde las características protectoras del hormigón son restablecidas y mantienen pasiva la superficie de las armaduras.

En el esquema de la Fig. 6 se puede notar cómo la elevada corriente requerida para una eficaz extracción de cloruros lleva al potencial de las armaduras al campo de posible desprendimiento de hidrógeno, por lo cual, la técnica no se puede aplicar si la polarización afecta a aceros de alta resistencia (hormigón pretensado).

\section{Estructuras carbonatadas}

En Fig.7 se muestra un esquema simplificado de la evolución de las condiciones en la superficie de una armadura embebida en un hormigón carbonatado y sometida a un tratamiento de realcalinización.

El punto 1 representa las condiciones en la superficie de la armadura hasta que el hormigón consigue su alcalinidad habitual. El punto 2 representa el momento en el cual el frente carbonatadoalcanza la armadura y el punto 3, una vez que se ha iniciado el ataque.

Si se aplica la protección catódica, la circulación de la corriente modifica las condiciones en la superficie de la armadura, llevándola del punto 3 al 4 . Consecuentemente, las armaduras se repasivan (nótese cómo el bloqueo de la corrosión no ocurre en este caso por la reducción del potencial, sino a causa de la realcalinización producida por la circulación de la corriente).

Considerando que la capa de hormigón afectada por estas

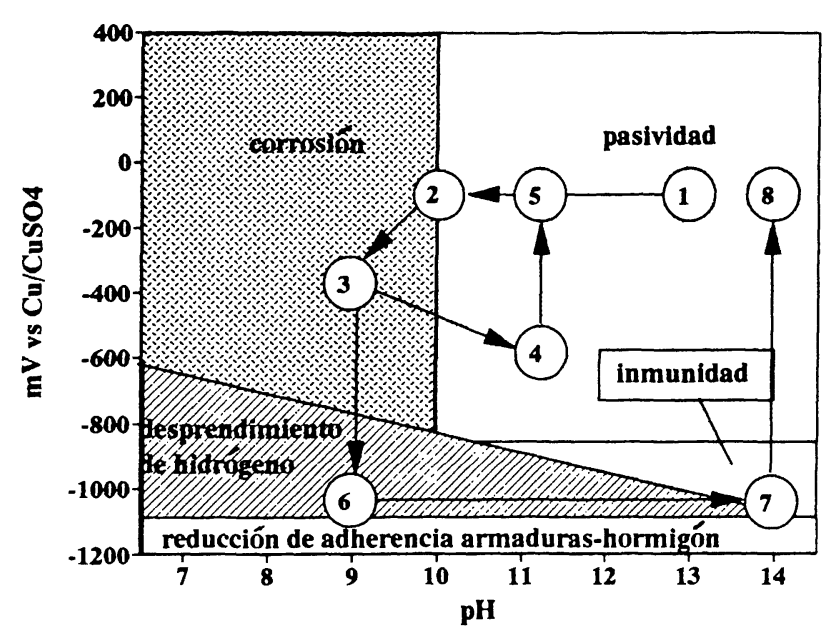

Fig. 7.- Condiciones de potencial y $\mathrm{pH}$ en la superficie de las armaduras durante un proceso de realcalinización electroquímica: condiciones de pasividad en hormigón sano (1); iniciación de la corrosión (2); antes de aplicar la realcalinización electroquímica (3); durante la polarización (67); y a tratamiento terminado (8). Para comparación se indican los puntos que representan la eventual aplicación (4) y posterior interrupción (5) de la protección catódica.

modificaciones permanece, de cualquier manera, muy delgada (1-2 mm), la interrupción de la corriente lleva, después de tiempos relativamente breves, de nuevo a la carbonatación y luego al ataque corrosivo.

De la realcalinización se puede repetir substancialmente lo que se ha dicho para la extracción de los cloruros. También se impone una corriente muy elevada (2-3 órdenes de magnitud más de la que se impone en la protección catódica), con relevante producción de alcalinidad en la superficie catódica. Por tanto, después de la aplicación de la corriente se pasa del punto 6 al punto $7 \mathrm{y}$ luego al punto 8 , antes $\mathrm{y}$ después de la interrupción de la circulación de corriente.

En el caso de la realcalinización, las modificaciones del hormigón afectan a un amplia región alrededor de las armaduras. En esta región, la disolución presente en los poros del hormigón alcanza valores de $\mathrm{pH}$ elevados $(>13)$ $\mathrm{y}$, por eso, las armaduras son repasivadas y permanecen también así una vez terminado el tratamiento, a diferencia de lo que pasa con la protección catódica. Sin embargo, puesto que la realcalinización afecta a la superficie de los poros del hormigón y a la disolución contenida en ellos, pero no a toda la masa del hormigón, una estructura realcalinizada con respecto a una nueva tiene una resistencia a la carbonatación menor. También en el caso de la realcalinización, debido a los potenciales que se alcanzan, existe la posibilidad de desprendimiento de hidrógenoy, en consecuencia, el riesgo de fragilización de los aceros de alta resistencia, por tanto, esta técnica, tampocose puede utilizar paraestructuras pre opostensadas. 


\section{LA PRÁCTICA DE LA REALCALINIZA- CIÓN}

Las condiciones en las que en la práctica se realiza el proceso son las siguientes:

-densidad de corriente, generalmente de $1-2 \mathrm{~A} / \mathrm{m}^{2}$, referida a la superficie de las armaduras;

-tiempo: de 3 días a 3 semanas;

-compartimento anódico externo al hormigón: constituido por una disolución alcalina de carbonato de sodio, que se renueva a medida que la acidez producida por la reacción tiende a reducir el $\mathrm{pH}$. Los ánodos empleados son frecuentemente a base de titanio activado.

Una vez que el hormigón cercano a las armaduras ha sido realcalinizado, aproximadamente un quinto de la alcalinidad que se ha producido se aplica en elevar la alcalinidad del hormigón que rodea las armaduras y los 4/5 que quedan en elevar la alcalinidad del hormigón adyacente a esta primera zona, que forma parte de lo que podemos llamar compartimento central. Se puede notar cómo en este compartimento la alcalinidad entra, pero prácticamente no sale. En consecuencia, el volumen de hormigón realcalinizado crece con el tiempo.

Naturalmente, la realcalinización concierne solamente al hormigón afectado por la circulación de corriente. En el caso de las geometrías mas usuales, la distribución de $\mathrm{pH}$ alrededor de las armaduras resulta la de la Fig.8.
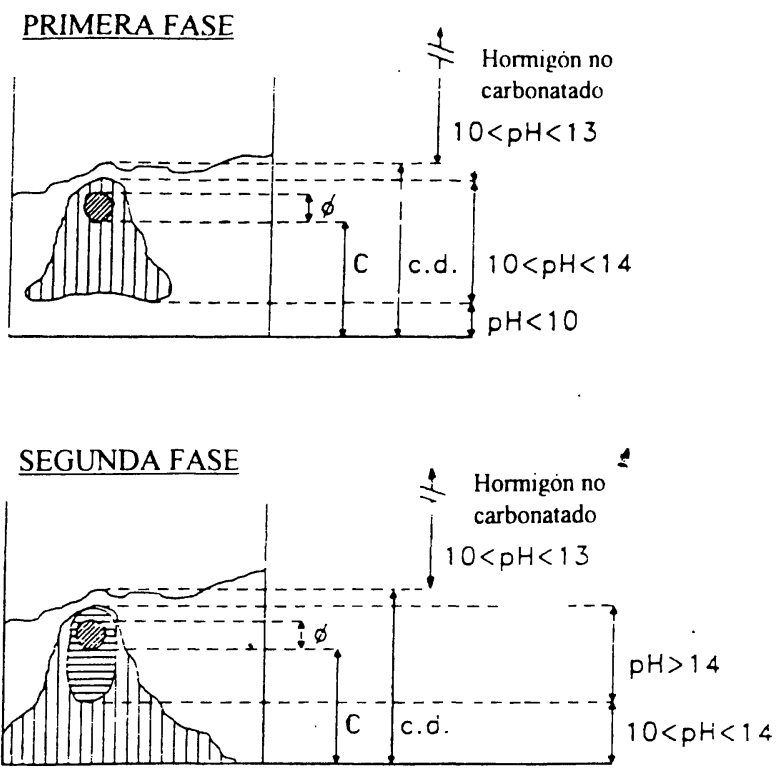

Fig. 8.- Extensión de la zona realcalinizada alrededor de la armadura a mitad del tratamiento (primera fase) y al término del tratamicnto (segunda fase); $\mathrm{C}=$ recubrimiento, $\mathrm{s} . \mathrm{c} .=$ espesor del estrato carbonatado [1]
La distribución de corriente en el interior del hormigón y en la superficie de las armaduras no es uniforme. En particular, en presencia de un doble estrato de armaduras, con las elevadas densidades de corriente del proceso, el primer estrato de armaduras absorbe hasta un $90 \%$ de la corriente total $[1,5]$. La consecuencia es que la realcalinización, de hecho, afecta sólo al recubrimiento.

\section{LA PRÁCTICA DE LA EXTRACCIÓN ELECTROQUÍMICA DE CLORUROS}

La técnica electroquímica de extracción de cloruros en las estructuras de hormigón contaminado por cloruros es muy parecida a la utilizada para la realcalinización: además, mientras que se extraen los cloruros, se restablecen también las condiciones de alcalinidad en las armaduras. La extracción de cloruros, sin embargo, requiere tiempos de aplicación sensiblemente más elevados de los necesarios para la realcalinización.

La posibilidad de aplicar esta técnica fue verificada por el Battelle Columbus Laboratories, en 1975 [6]. Se ejecutaron los ensayos sobre probetas aplicando $100 \mathrm{~V}$, por un período de 12-24 horas, utilizando redes de titanio platinado como ánodo e hidróxido de calcio como electrólito. Se utilizó también una resina de intercambio iónico para limitar el desprendimiento de cloro. Trabajos posteriores demostraron que era necesario aplicar, por lo menos, $0,46 \mathrm{~A} / \mathrm{m}^{2}$.

Se desarrolló también un ensayo de campo sobre una pequeña sección de un puente en Ohio [4], con resultados prometedores. Un ensayo paralelo fue llevado a cabo en los mismos años por el Kansas Department of Transportation, que utilizó una tensión de $220 \mathrm{~V}$ y corrientes de $22 \mathrm{~A} / \mathrm{m}^{2}$ y ánodo de cobre. Además de la elevada temperatura alcanzada, el efecto más negativo fue un considerable aumento de la porosidad del hormigón.

En Europa se han efectuado trabajos experimentales que han llevado a la comercialización del proceso Norcure, puesto a punto a mediados de los 80 por la sociedad noruega Norsk Overflate Teknik (NOT).

Parámetros típicos del proceso de la extracción de cloruros son:

-tensión aplicada: $40 \mathrm{~V}$ máximo

-densidad de corriente: generalmente 1-2 $\mathrm{A} / \mathrm{m}^{2}$ referidas a la superficie de las armaduras;

-tiempo: de tres semanas a tres meses;

-compartimento anódico exterior: ánodos a base de titanio activado, sumergidos en una disolución saturada de hidróxido de calcio. 
Hasta ahora se han tratado con el proceso Norcure aproximadamente $40.000 \mathrm{~m}^{2}$ de hormigón armado, pero sólo unos pocos proyectos tienen amplia documentación de los resultados obtenidos. Entre estos últimos figura un proyecto llevado a cabo en Estados Unidos y Canadá con el soporte SHRP (Strategic Highway Research Program) y uno en Suiza bajo control de la Politécnica Federal de Zurich $[11,12]$.

En la Fig.9 se indican algunos datos experimentales que hacen referencia a la extracción de cloruros al variar el tiempo de aplicación, obtenidos en los ensayos americanos.

La Fig. 10 muestra la evolución de la tensión aplicada y de la corriente en el caso del proyecto suizo. La reducción de la corriente durante el tratamiento es debidaal aumento de la resistencia del hormigón. Esta disminución contribuye, junto a la disminución de los cloruros y de su número de transporte, a reducir la eficiencia del proceso a medida que éste avanza.

Los experimentos seguidos por la Politécnica de Zurich muestran una reducción del total de los cloruros parecida,

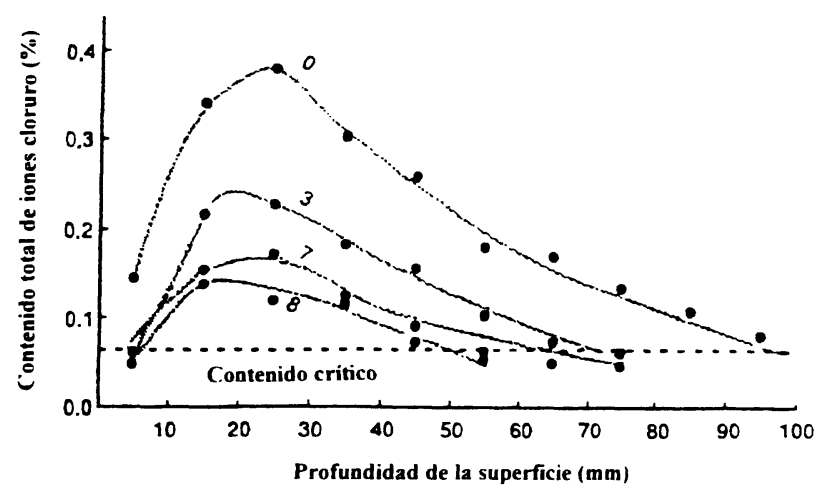

Fig. 9.-Perfil de concentración de cloruros (en relación al peso dehormigón) después de $0,3,7,8$ semanas de tratamiento [7].

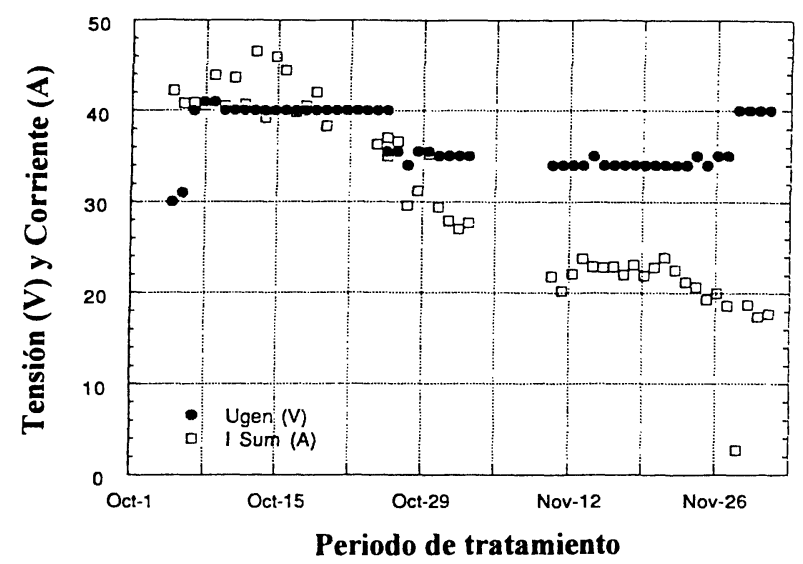

Fig. 10.- Evolución de la tensión (U) y de la corriente (I) durante el tratamiento de extracción electroquímica de cloruros de un muro de, aproximadamente, $60 \mathrm{~m}^{2}[11]$ es decir, comprendida entre el $50 \%$ y $60 \%$. Estos ensayos han sido efectuados sobre un muro, caracterizado por una penetración de los cloruros muy heterogénea. Para la extracción de los cloruros en las zonas de más contaminación, ha sido necesario un doble tratamiento. Los mapas de potencial, efectuados antes del tratamiento, mostraban que, aproximadamente el $30 \%$ de las armaduras, estaban sometidas a corrosión; mientras que los efectuados después del segundo tratamiento (que muestran potenciales $80-100 \mathrm{mV}$ mas positivos) indican la repasivación de todas las armaduras (Fig.11).

Para las estructuras contaminadas por cloruros valen, por lo que concierne a la distribución de la corriente, las mismas consideraciones hechas para las estructuras carbonatadas. Por lo tanto, la extracción de cloruros, como la realcalinización, de hecho afecta solamente al recubrimiento.

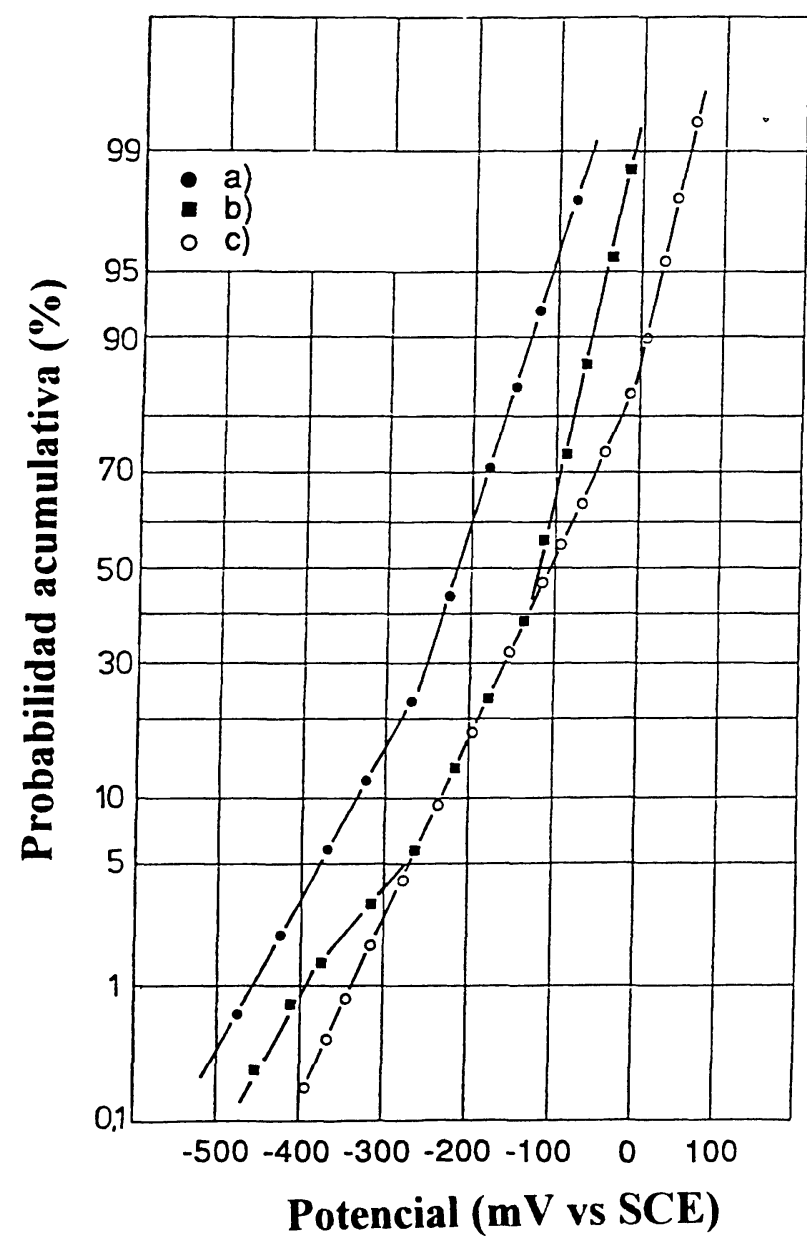

Fig. 11.- Probabilidad acumulativa de los potenciales de las armaduras de un muro sometido a extracción de cloruros: a) antes del tratamicato; b) después del primer tratamiento; c) después del segundo tratamiento. I as medidas b) y c) sehan efectuado, aproximadamente, 6 meses después de los respectivos tratamientos $[11,12]$. 


\section{LOS EFECTOS SECUNDARIOS}

La aplicación de estas técnicas electroquímicas produce algunos efectos secundarios, algunos de los cuales no deseados y, en algunos casos, peligrosos. Entre ellos se destacan:

Fragilización por hidrógeno. Si están presentes armaduras de acero de alta resistencia, como en el caso de estructuras pretensadas o postensadas, estas técnicas no se pueden aplicar. En efecto, las altas densidades de corriente que se utilizan provocan desprendimiento de hidrógeno en las superficies de las armaduras y causan en aquellos aceros de elevadas características mecánicas (límite elástico $\sigma>1.000 \mathrm{MPa}$ ) fenómenos de fragilización por hidrógeno.

Reacción árido-álcali. Algunos tipos de áridos son químicamente inestables en presencia de elevados contenidos de álcalis en el hormigón y pueden reaccionar con estos produciendo un gel que puede absorber agua y expandirse. $\mathrm{El}$ resultado de la reacción álcali-árido es un aumento de volumen, que puede llevar a la disgregación o a la pérdida de características mecánicas del hormigón.
Ya que la aplicación de las dos técnicas electroquímicas conlleva, en el cátodo, tanto el desarrollo de elevados valores de $\mathrm{pH}$ como la migración de álcalis $\left(\mathrm{Na}^{+} \mathrm{y} \mathrm{K}^{+}\right)$, es posible suponer un significativo aumento de los efectos expansivos en la proximidad de la armadura en el momento en el que se apliquen la realcalinización o la extracción electroquímica de los cloruros a estructuras con áridos potencialmente susceptibles a la reacción árido-álcali. Recientes estudios confirman esta hipótesis $[2,10,14]$, por lo que es absolutamente desaconsejable la aplicación de estas dos técnicas a estructuras que se piensa puedan ser susceptibles a este tipo de ataque.

Pérdida de adherencia. El empleo de elevadas densidades de corriente, necesarias para las exigencias prácticas de reducción de los tiempos de aplicación, puede llevar a un daño del hormigón en contacto con las armaduras, con la consecuente pérdida de adherencia. Se puede atribuir la causa principal de este daño al desprendimiento de hidrógeno; también otras posibles causas de disminución de adherencia, actualmente en fase de estudio, son los efectos de una reacción árido-álcali o la debilitación de la pasta de cemento, causada por la acumulación gradual de iones $\mathrm{Na}^{+}$y K $\mathrm{K}^{+}$en la proximidad de la armadura $[2,15]$.

\section{BIBLIOGRAFÍA}

[1] R.POLDER, H.J.VAN DER HONDEL: "Elechtrochemical Realkalinization and Chloride Removal of Concrete" Proc. of RILEM Conference "Rehabilitation of Concrete Structures" pp. 135-147, Melbourne, 1992.

[2] C.L.PAGE: "Interfacial Effects of Electrochemical Protection Method Applied to Steel in Chloride Containing Concrete" Proc. of RILEM Conference "Rehabilitation of Concrete Structures" pp. 179-188, Melbourne, 1992.

[3] P.PEDEFERRI: "La corrosione delle armature nel calcestruzzo" en "Corrosione e protezione delle strutture metalliche negli ambienti naturali e nel cemento armato", CLUP, Milano (1987), pp. 419-479.

[4] J.E.BENNET, T.J.SCHUE: "Electrochemical chloride Removal from Concrete: a SHRP Contract Status Report", Corrosion/90, paper n.316, NACE, Houston, TX, 1990.

[5] T.PASTORE, P.PEDEFERRI, L.BERTOLINI, F.BOLZONI: "Current Distribution Problems in the Cathodic protection of Reinforced Concrete Structures", Proc. of RILEM Conference "Rehabilitation of Concrete Structures" pp. 189-200, Melbourne, 1992.

[6] B.ISECKE, J.MIETZ: “Investigation on Realkalinization of Carbonated Concrete”, Eurocorr '91, Budapest, 1991, pag.732-738.

[7] D.G.MANNING: "Electrochemical Removal of Chloride Ions from Concrete", Zurich, SIA Dokumentation D065, 1991, pag.6168.

[8] L.LAZZARI: "Rialcalinizzazione, rimozione dei cloruri, iniezione di inibitori", Actos del curso de Istruzione Permanente de Politecnico di Milano sulla "Corrosione delle armature nelle strutture in c.a.", Politecnico di Milano, Giugno 1992.

[9] M.F.ASARO, A.T.GAYNOR, S.HETTIARACHI: "Electrochemical Chloride Removal and Protection of Concrete Bridges Components (Injection of Synergistics Corrosion Inhibitors)", SHRP Report No. S/FR-90-002, 1990.

[10] G.SERGI, C.L.PAGE, D.M.THOMPSON: "Electrochemical induction of alkali-silica reaction in concrete", Materials and Structures, 24, 1991, pag.359-361.

[11] B.ELSENER, M.MOLINA: “Elektrochemische Chloriddenfernung an Stalbetonbauwerken”, EVED Bundersamt fur Strassenbau, Bern (1992). 
[12] B.ELSENER, M.MOLINA, H.BOHNI: "The Electrochemical Removal of Chlorides from Reinforced Concrete", Corrosion Science, 35, 1993, pag. 1536-1570.

[13] P.PEDEFERRI: "Protezione e prevenzione catodica delle armature nelle costruzioni in c.a. e c.a.p.", L'Edilizia 7, 10, pag. 69- . 81, 1993.

[14] C.L.PAGE, S.W.YU: "The effect of Chloride Removal on Alkali-Silica reaction", SCI conference "Electrochemical Repair of Reinforced Concrete", London, 16 September 1993.

[15] N.BUENFELD: "Effect of Chloride Removal on Rebar Bond Strenght and Concrete Properties", SCI conference "Electrochemical repair of reinforced Concrete", London, 16 September 1993.

[16] C.ANDRADE: "Calculation of chloride diffusion coefficients in concrete from ionic migration measurements", Cement and Concrete Research, 23, 1993, pag.724-742

[17] C.ALONSO, C.ANDRADE: “Corrosión de las armaduras de acero debida a la carbonatación acelerada de soluciones que simulan la composición de la fase acuosa del hormigón”, Materiales de Construcción 37, n²06, abril-junio 1987, pag.5-14.

\section{Publicaciones del Instituto Eduardo Torroja - CSIC}

Número monográfico de MATERIALES DE CONSTRUCCIÓN

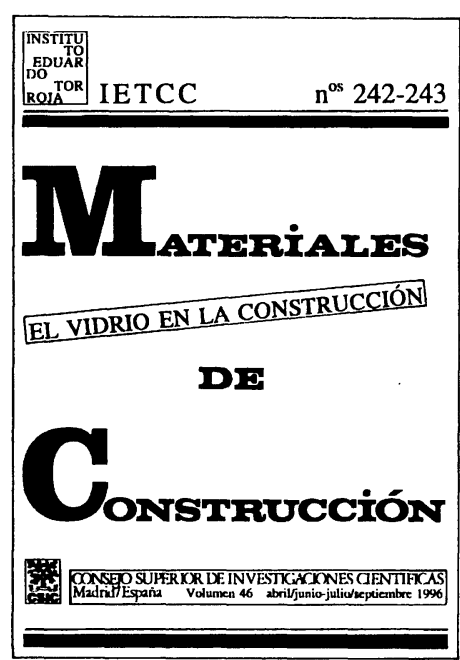

EL VIDRIO EN LA CONSTRUCCIÓN

$\left(n^{\text {os }} 242-243\right)$
- Procesos de alteración de las vidrieras medievales. Estudio y tratamientos de protección. (Alteration processes of medieval stained glass windows. Study and protection treatments). J. M Fernández Navarro.

- El efecto de la corrosión en vidrieras coloreadas. (The effect of corrosion of stained glass windows).

J. I eissner.

- Formulación de vidrios absorbentes del calor.

(Formulation of heat absorbing glasses).

P. Álvarez-Casariego y P. Mazón.

- Dimensionamiento de placas de acristalamiento para edificios mediante un modeloprobabilístico de rotura. (A probabilistic model for failure design of glass plates in building).

A. Fernández Canteli. I. Viña y A. Bernardo Sánchez.

- Caracterización de fibras en forma de lana de roca para aislamiento obtenidas a partir de basaltos canarios. (Characterization of fibers as rockwool for insulation obtained from canary islands basalts).

J. M. Cáceres, J. E. García Hernández y J. M Rincón.

- El GRC: Material compuesto de matriz inorgánica reforzado con fibras de vidrio AR.

(GRC: Composite material from an inorganic matrix reinforced with ar glass fibres).

P. I. Comino.

- Los materiales vitrocerámicos en la construcción.

(Glass-ceramic as building materials).

J. MP Rincón y M. Romero.

- Gres porcelánico: Aplicaciones arquitectónicas, procesado y propiedades físico-mecánicas.

(Porcelainized stoneware: Architectural, processing and physico-mechanical properties).

T. Manfredini, M. Romagnoli y J. Mª Rincón.

Venta de ejemplares: distribución de Publicaciones

Instituto de Ciencias de la Construcción Eduardo Torroja

c/Serrano Galvache, s/n - 28033 Madrid

Tfno..(91)302.04.40-Fax:(91)302.07.00 